\title{
Application of sequential spatial clustering and fractal dimension to caving seismic event parameters of time, distance, and intensity
}

\author{
DM Cortolezzis Laurentian University, Canada \\ MR Hudyma Laurentian University, Canada
}

\begin{abstract}
Recent research has developed a seismic event clustering method that groups seismic events spatially while preserving the event sequence. The main benefit of this method is that it can be done proactively at any point in time as seismicity progresses. Since caving is a progressive non-blasting mining method, this clustering method could potentially detect changes in the progression of the rock mass as they occur and without retroactive analysis.
\end{abstract}

This paper demonstrates the use of the new method using data from a caving mine. In particular, the fractal dimension of the seismic source parameters of time, distance and intensity are used to characterise the seismic events during three periods: development of the undercut, cave initiation, and cave propagation. The data will become a benchmark case study characterising a caving rock mass.

Keywords: caving, sequential, clustering, mining-induced seismicity, fractal dimension

\section{Introduction}

Mines using caving as a mining method need to know the location and extent of fracturing induced by the caving process in order to keep workers safe from airblasts (Vejrazka 2016) and ensure adequate broken material and grade for production. Cave monitoring techniques usually include microseismic monitoring and analysis, deep-hole extensometers, open boreholes, video surveys, and visual observations of pre-existing excavations (Digiovinazzo \& Singh 2010; Pfitzner 2003).

In the study of seismic data from the Palabora mine (Glazer 2012), all the cave seismic events and seismic activity to estimate hazard are analysed as one unit or one large cluster. While this approach provides good overall information, it cannot identify specific areas within the cave. Clustering of all seismic events in and around a cave provides specific information about different areas within the caving zone. This information is important, particularly to monitor cave progression by the shape of the cave seismic events. In Pinnock et al. (2016) two clusters of events were processed using a collapsing method (Jones \& Stewart 1997) to see if the events were part of a structure. The collapsing method uses an algorithm to determine the error ellipsoid of each individual event and then moves neighbouring events to a common point to help determine if they are random events or events from the same structure. A simpler method to achieve the same results is desirable.

Mining-induced seismicity has been observed to cluster in space and over time (Trifu et al. 1993; Kijko \& Funk 1996). A particular difficulty with previous spatial-temporal studies of seismicity is that a study time frame must be chosen (Kijko et al. 1993; Eneva \& Villeneuve 1997; Lesniak \& Isakow 2009). The time frame chosen (a week, month, year, etc.) is imposed on the data and the results of space-time clustering can vary drastically based on the time frame chosen. These types of clustering methods are unable to preserve the sequence which inhibits temporal study of clusters. By clustering spatially and sequentially, the entire life of a cluster can be studied without having to choose a study time frame. 
Using clustering as an analysis technique for mining-induced seismicity was brought to the forefront in a two-step process described in Hudyma and Potvin (2008). The method was then applied to the cave at the Ridgeway mine (Potvin \& Hudyma 2008). This work was an important first step in applying clustering to mining seismic data, particularly caving seismicity, to investigate seismic sources. Further work in clustering methodology has recently been summarised in Woodward et al. (2018). The paper is a thorough and useful discussion of clustering methods. In particular, this paper provides a number of points outlining why density-based clustering is well-suited to mining-induced seismicity.

This paper will describe a novel density-based clustering method called sequential spatial clustering. The method is a one step, unsupervised method that is a logical extension of the work by Hudyma \& Potvin (2008). The method clusters events one at a time which preserves the sequence in which the events occur. The significance of this feature is that time and location can be studied together for each cluster in order to provide new insight into cave movement. The sequential spatial clustering method also has the ability to show the shape and size of a cluster at any point in time without additional data processing.

There are other significant benefits to the new method. First of all, it uses all seismic events in a dataset, removing the need to select which events to include. Choosing which seismic events for analysis is typically done by visually selecting events that appear to be in the area of interest, examples of which are shown in de Beer et al. (2017) and Pinnock et al. (2016). The distance used to cluster events is derived from the distribution of nearest neighbour (NN) distances for each event at the time the event occurs. Thus, the clustering distance is based on what the data reveals about itself and is not an arbitrarily chosen distance. Events that are not close to any other event remain as single isolated events unless another event occurs close to it at some point in time. Each cluster and single isolated event identifies seismically active locations. This means that additional seismic events could be expected at any of these locations. Since clustering takes place one event at a time, the sequence of each cluster is preserved and can be studied at any point in time, removing the bias created by time windows. A final benefit is that the method can be used with as many or few events as are available.

Sequential spatial clustering was first developed using seismic sources of a failing pillar, a developing ramp, stope abutments, and a shear zone (Cortolezzis 2018). These case studies were instrumental in showing that the method gives new insight into inaccessible seismically active locations which is a primary need in cave mining (Pfitzner 2003). This novel clustering method could prove useful to mines that use caving mining methods (sublevel, block, and panel) by providing a new perspective to the location of the cave back at any point in time, particularly if cave propagation stalls or changes direction.

\section{Methodology}

\subsection{Clustering}

In order to cluster seismic events, the distance between events must be calculated. A cluster is created when events that are within a chosen clustering distance are grouped together. The choice of which events to cluster and at what distance must be reflective of the data itself and should not be arbitrarily chosen. The sequential clustering method uses all true seismic events in a dataset; noise from equipment or orepasses and blast events are removed. The following section describes how the data is used to determine the clustering distance.

\subsubsection{Distribution of nearest neighbour distances}

In this methodology, nearest neighbour distances of previous events are used to determine whether or not a seismic event joins a cluster. This is accomplished by calculating the distance between each seismic event and every other prior seismic event. The event that is closest to the most recent event is the nearest neighbour and the distance between them is the nearest neighbour distance. Previous research has shown that distribution of nearest neighbour distances has a lognormal distribution (Cortolezzis 2018). Figure 1 shows the distribution of the nearest neighbour distances of 3,704 seismic events which have a lognormal 
distribution. The mode and mean nearest neighbour distances are used to cluster the events rather than arbitrarily choosing a distance. These distances are a direct reflection of the seismic data and the method is reproducible for any size population of nearest neighbour distances.

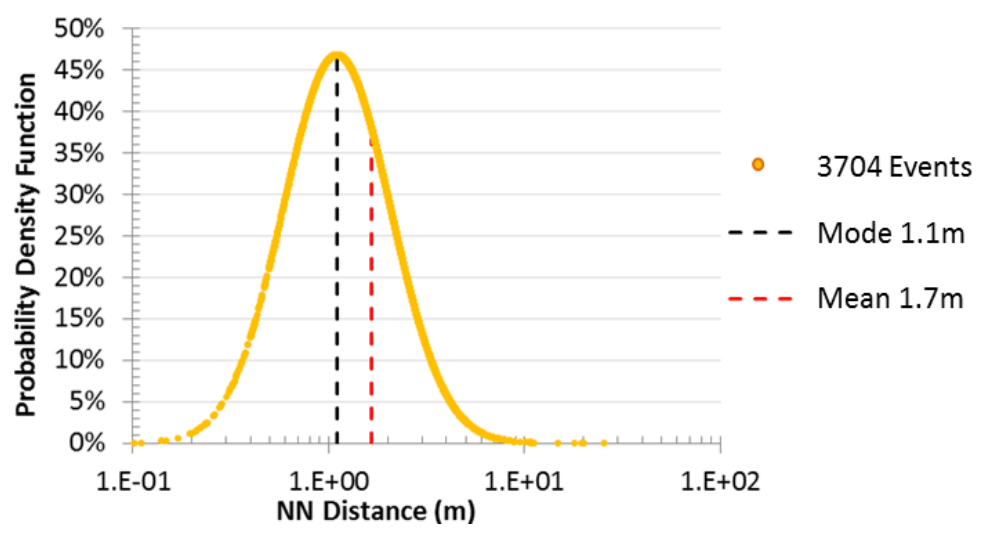

Figure 1 The lognormal distribution of the nearest neighbour distances for 3,704 seismic events

The mode nearest neighbour distance (1.1 $\mathrm{m}$ in Figure 1) identifies the clusters and locations that are most seismically active. The mean nearest neighbour distance $(1.7 \mathrm{~m})$ gives a good indication of cluster size (number of events) and its shape (Cortolezzis 2018).

\subsubsection{Cluster process}

Once the clustering distance has been determined, clusters can be created one event at a time. While the progression of sequential spatial clustering could be shown in 20 images, it has been condensed to four images in Figure 2 (images $A$ to $D$ ). The status of the clusters and single events will be described. Image $A$ shows the first 20 events from the seismic data population shown in Figure 1 before the clustering technique has been applied. When looked at as a group, it is difficult to determine which events are related to each other. If these events were analysed as a group, it would be done with the assumption that the events are all related without any evidence to support the assumption.
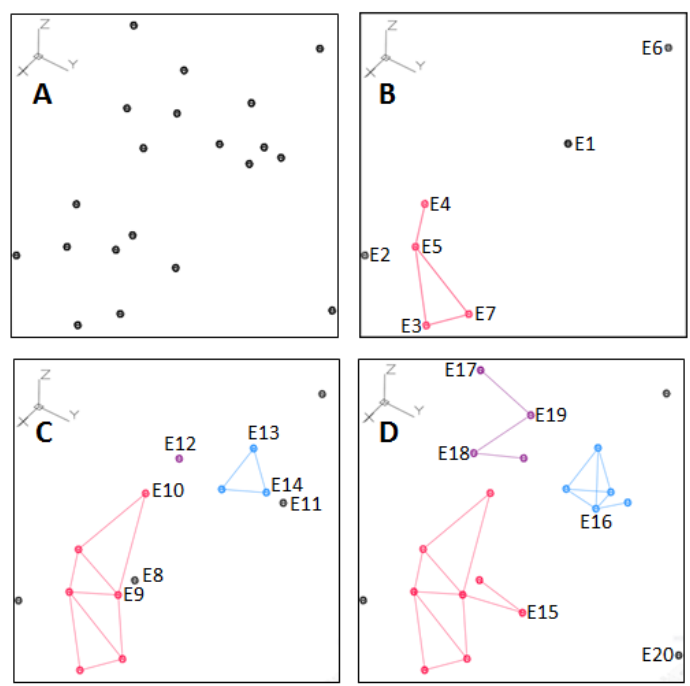

Figure 2 Image $A$ is an isometric view of twenty seismic events. Images $B, C$, and D show the progress of cluster formation after 7,14 , and 20 events. A line is drawn when an event is within $1.7 \mathrm{~m}$ of any preceding event

Image B shows how a cluster forms after the first seven events. In this image, events $1,2,3$, and 4 are single events when they occur. Event 1 does not have a nearest neighbour and events 2 through 4 are not within $1.7 \mathrm{~m}$ of any preceding event. Event 5 is within $1.7 \mathrm{~m}$ of events 3 and 4 , so lines are drawn to show these 
relations. Event 6 is not close enough to any preceding event and also remains a single event. Event 7 is within $1.7 \mathrm{~m}$ of events 3 and 5 , so lines are added. At this point, that is when event 7 occurs, one cluster has formed that is comprised of four events $(3,4,5$ and 7$)$, and three events remain as single events $(1,2$ and 6$)$.

Image $\mathrm{C}$ shows the status of the clusters and single events after 14 events. At this point, two clusters have formed. The first cluster has six events $(3,4,5,7,9$ and 10$)$ and the second cluster has three events $(1,13$ and 14). It can been seen at this stage that the size of the first cluster has increased from four events to six events and the shape is more complex in Image $C$ than in Image $B$. There are five events which remain as single, isolated events $(2,6,8,11$ and 12$)$.

When sequential spatial clustering of all 20 events has been applied (Figure 2 Image D), it can be seen that three clusters of different sizes form: red (eight events), blue (five events) and purple (four events). The shapes are also different with the purple cluster being linear, the blue cluster a tetrahedron with a linear arm, and the red cluster as a plane with a linear arm.

Figure 2 Image A shows the same twenty events as Image D. In Image A, it is difficult to discern which events are related by distance, while Image $D$ shows how events are related by their nearest neighbour distances when the sequential spatial clustering method is applied. In Image $D$, the size and shape of each cluster reflects the seismic activity at that location. The three events that remain as single isolated events may be indicators of other locations that may become seismically active in time.

While the cluster process uses the sequence of events to form clusters, each event occurs at a specific time, and the time evolution of each cluster can be seen by replacing the sequence number with the corresponding time of that event.

\subsection{Fractal dimension}

The use of fractal dimension as a statistical method to study mining-induced seismicity was introduced in the 1990s (Coughlin \& Kranz 1991; Eneva \& Young 1993; Xie \& Pariseau 1993; Trifu et al. 1993; amongst others). It is often used on complex systems or processes, such as seismicity, that have non-Euclidean geometry and are scale invariant (Mandelbrot 1982). This provides a means of quantifying the system or process that otherwise could not be explained. As the focus of the research at that time was to develop a method to forecast rockbursts, limitations in the application of fractal dimensions existed. For example, inter-event distances were used, which makes the assumption that all events are related when they may not be. The nearest neighbour distances used in this paper do not make the same assumption. Instead, it relies on the distribution of the nearest neighbour events to determine nearest neighbour distance relations. Nearest neighbour events do have ranges and are directly related to the physical process. Fractal dimension mathematics can be applied to any dataset where a relation is sought. In this paper, it will be applied to nearest neighbour distances and time between nearest neighbours. The fractal dimension of seismic event intensity is calculated by relating a change in nearest neighbour distance for each magnitude range and by the time between nearest neighbours by magnitude range.

While there are a number of ways to calculate fractal dimension (Klinkenberg 1994), the correlation integral method is used most often as it does not require a large dataset, nor does it introduce a bias based on an input parameter. The use of fractal dimension to seismic databases did not continue after the 1990s work and was largely hampered not by the application of fractal math but by the seismic databases that contained noise, blasts and/or multiple seismic source mechanisms. The use of these types of seismic databases often led to inconclusive or conflicting results. The interest in applying fractal math to rock masses has been pursued again by recent work by Li et al. (2017). In this research, fractals were used to show the scale effect of surface roughness for natural surfaces. It was found that joint surfaces were not infinitely fractal but existed in limited scales and that the application of fractals provided a useful framework for interpreting surface morphology. One main difference between fractal surfaces and seismicity is that the profile of a natural surface is self-affine while seismicity is self-similar. 


\subsubsection{Correlation integral}

The correlation integral described by Hirata et al. (1987) is shown in Equation 1 and is used to determine the fractal dimension of the distance between nearest neighbour events.

$$
C(R)=\frac{n(r<R)}{P}
$$

where:

$$
\begin{array}{ll}
C(R) & =\text { correlation integral. } \\
r<R & =\text { nearest neighbour distance up to the clustering distance. } \\
P & =\text { pair ratio (number of nearest neighbour pairs } \times \text { number of events minus } 1 \text { ). } \\
n(r<R) & =\text { \# of pairs. } \\
R & =\text { clustering distance. }
\end{array}
$$

A relation of the parameter being studied is fractal if a plot of $\log C(R)$ versus $\log R$ results in a straight line. This means $n(r<R)$ is proportional to the power function of $R$.

\section{Data}

This dataset contains 13,918 events that cover a 21 month period when the undercut was developed, cave initiated and the start of cave propagation. The undercut was blasted over a 10 month period $(2,894$ seismic events), followed by the initiation of the cave (7,903 events), then one month of cave propagation $(3,121$ events). All seismic events resulting from rock mass failures are used with non-seismic events removed (orepass noise or blasts appearing as seismic events). The error in the locations was tested and did not have a material effect on the nearest neighbour distances. Figure 3 shows all 13,918 events.

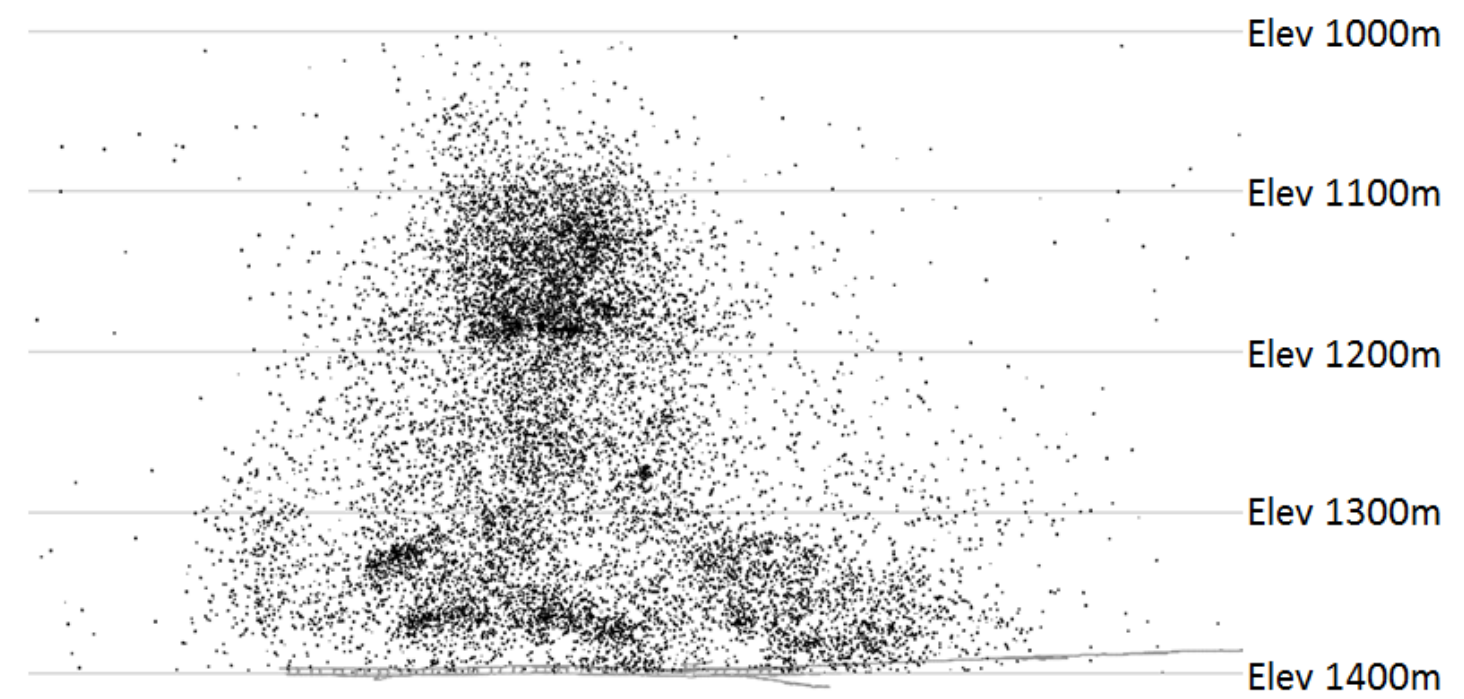

Figure 3 A side view of the 13,918 seismic events that occurred over a 21 month period. While some areas appear denser than others, it is difficult to determine which events are related to each other 


\section{$4 \quad$ Results}

All of the events were clustered using sequential spatial clustering and then characterised by a fractal dimension and the range over which the fractal dimension exists. The results of each step of the process will be described.

\subsection{Determination of nearest neighbour distances}

The clustering distance for each phase of the cave is determined by the distribution of seismic events during each period. Figure 4 shows the probability density function for each of the three phases.

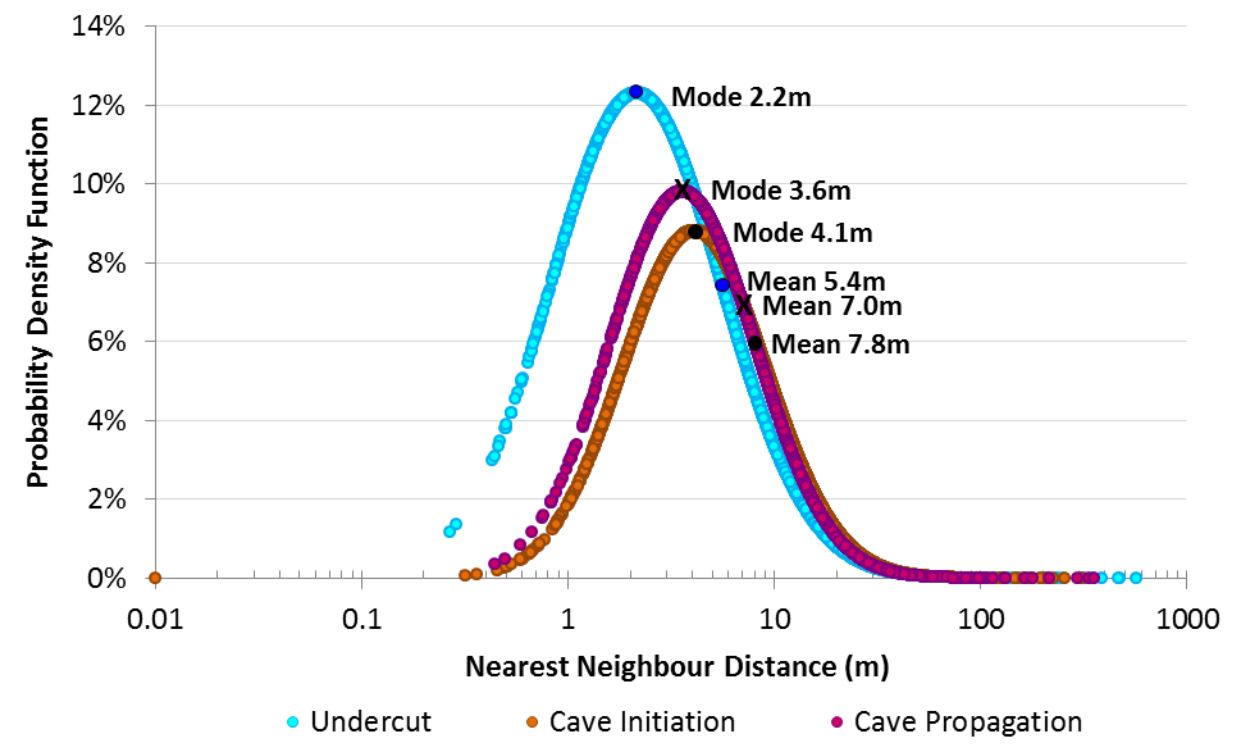

Figure 4 The distribution of nearest neighbour distances for each of the three periods: undercut, cave initiation, and cave propagation. There is some overlap between the time the undercut was developed and the cave initiated. Cave propagation started after the undercut was complete

The distributions of the nearest neighbour distances for each phase are distinctly different as blasting and the rate of production affect the rate of cave initiation and propagation. Each phase has a different mode and mean nearest neighbour distance, as well as differences in the range of minimum and maximum distances. The undercut nearest neighbour events are much closer together than the other two phases. This is expected in areas where blasting takes place. The cave initiation and propagation phases are similar, however, the initiation phase has a smaller range of nearest neighbour distances than the propagation phase.

\subsection{Sequential spatial clusters}

Each phase (undercut development, cave initiation and cave propagation) is clustered using the mean nearest neighbour distances so that the relative size of the phase can be seen. Figure 5 shows the number and size of clusters and remaining single isolated events for the undercut when the mean nearest neighbour distance of $5.4 \mathrm{~m}$ is used. At this point in time, the single isolated events are not related to any other event. As the rock mass continues to fail, some of these events will become part of a cluster if they are within the failing rock mass. The events that remain as single events after the cave breaks through to surface are indicative of a location that was seismically active only once. 


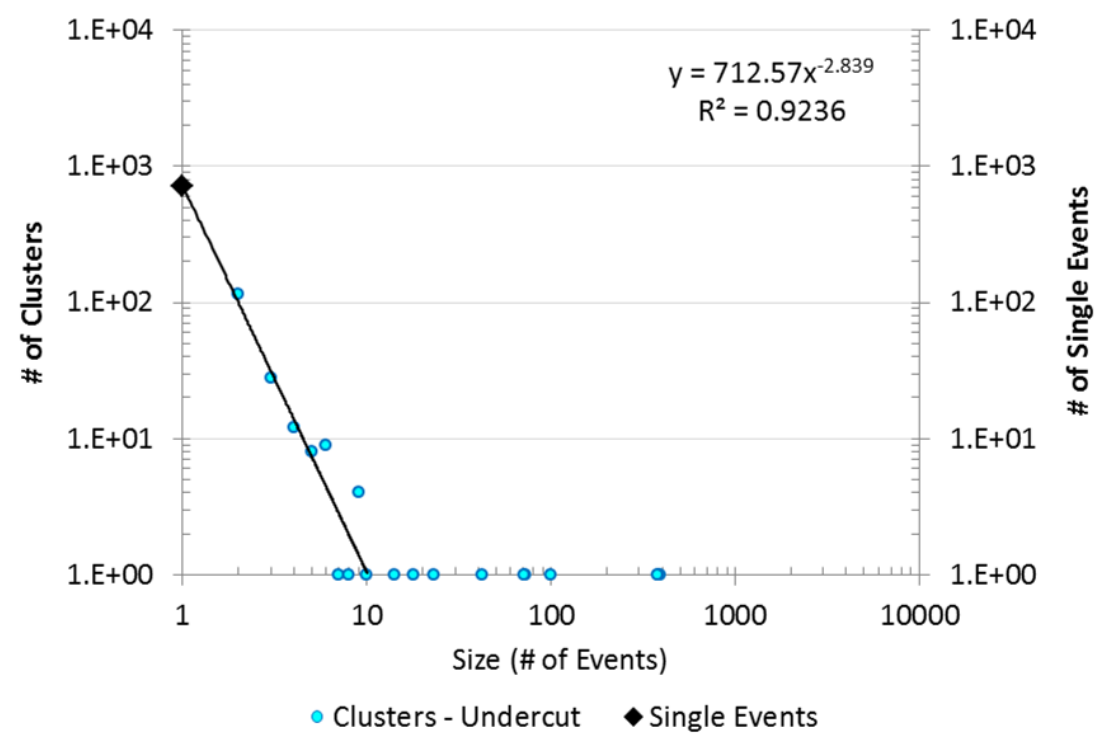

Figure 5 Cluster sizes formed during the development of the undercut using the mean nearest neighbour distance $(5.4 \mathrm{~m})$ to cluster the events

The number and size of clusters are characteristic of the undercut development phase. Most of the events are in small clusters of less than 10 events with a small number of clusters forming with less than 100 events. There is one large cluster of 340 events. Figure 6 shows the clusters in a side view above the undercut. The largest clusters are shown by different colours.

Elev $1000 m$

Elev 1100m

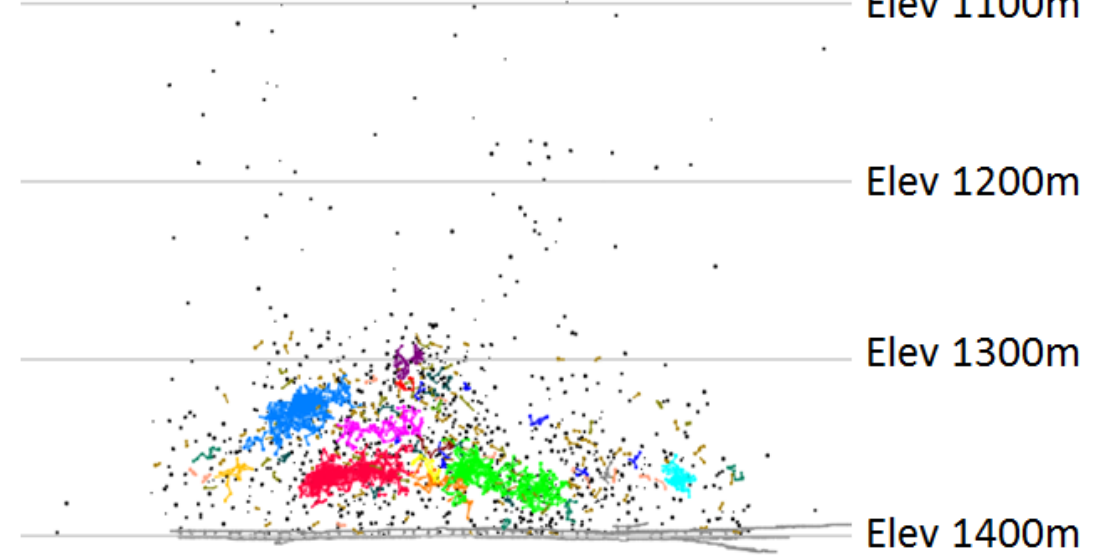

Figure 6 Side view of clusters above the undercut. Single isolated events occur over $350 \mathrm{~m}$ above the undercut, while the majority of the events and all the clusters are within $100 \mathrm{~m}$

With a closer look at the largest clusters in Figure 7, it can be seen that the clusters have various shapes and seismic event densities. Since the seismic events are locations where rock mass fracturing has taken place, it can be seen that the rock mass above the undercut has fractured differently in various locations. The cluster shown by the pink colour tends to have events fairly evenly distributed. In contrast, the darker blue cluster is larger, and has one obvious area where a large number of seismic events has occurred. 


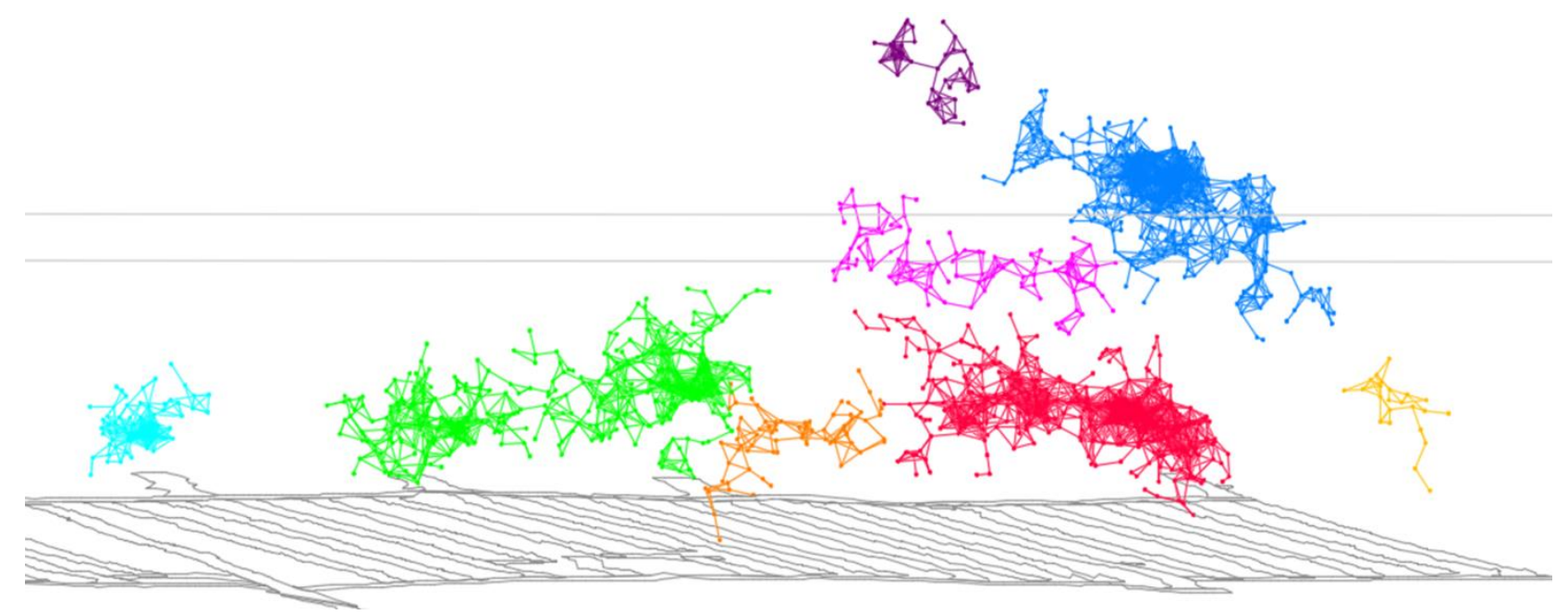

Figure 7 The eight largest clusters above the undercut after clustering with a nearest neighbour distance of $5.4 \mathrm{~m}$

Of interest are the areas where either no clusters or very few events have occurred. The rock mass may have fractured aseismically, or not have fractured sufficiently, which could cause problems at a later time. Since the data can be clustered at any point in time, areas that may be aseismic could be investigated as soon as they are identified so that proactive measures can be taken if necessary.

The cave initiation phase resulted in one large cluster of 1,693 events along with many smaller clusters around it when the mean nearest neighbour distance of $7.8 \mathrm{~m}$ was used (Figure 8).

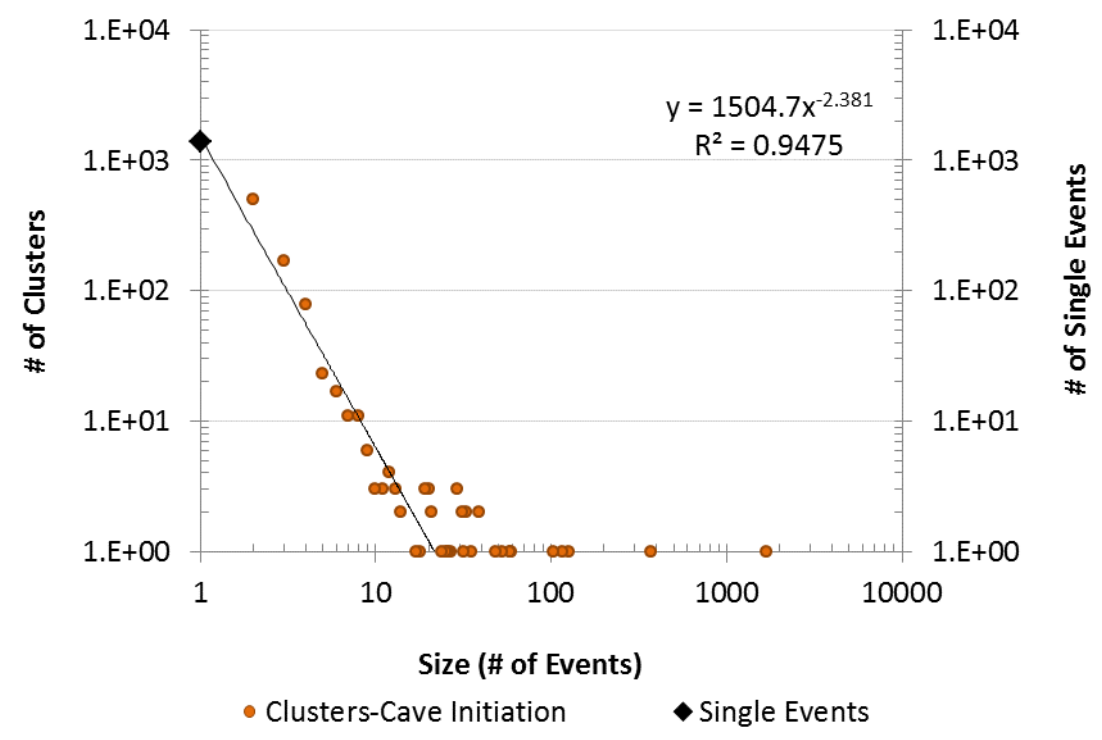

Figure 8 Clusters formed during the initiation of the cave. The mean nearest neighbour distance of $7.8 \mathrm{~m}$ is used to cluster the events

A significant characteristic of the cave initiation phase is a nearest neighbour distance that is larger than that of the undercut. In addition, the green cluster is at least 10 times larger than all the other clusters, with the exception of the light blue cluster (Figure 9). 
Elev 1000m

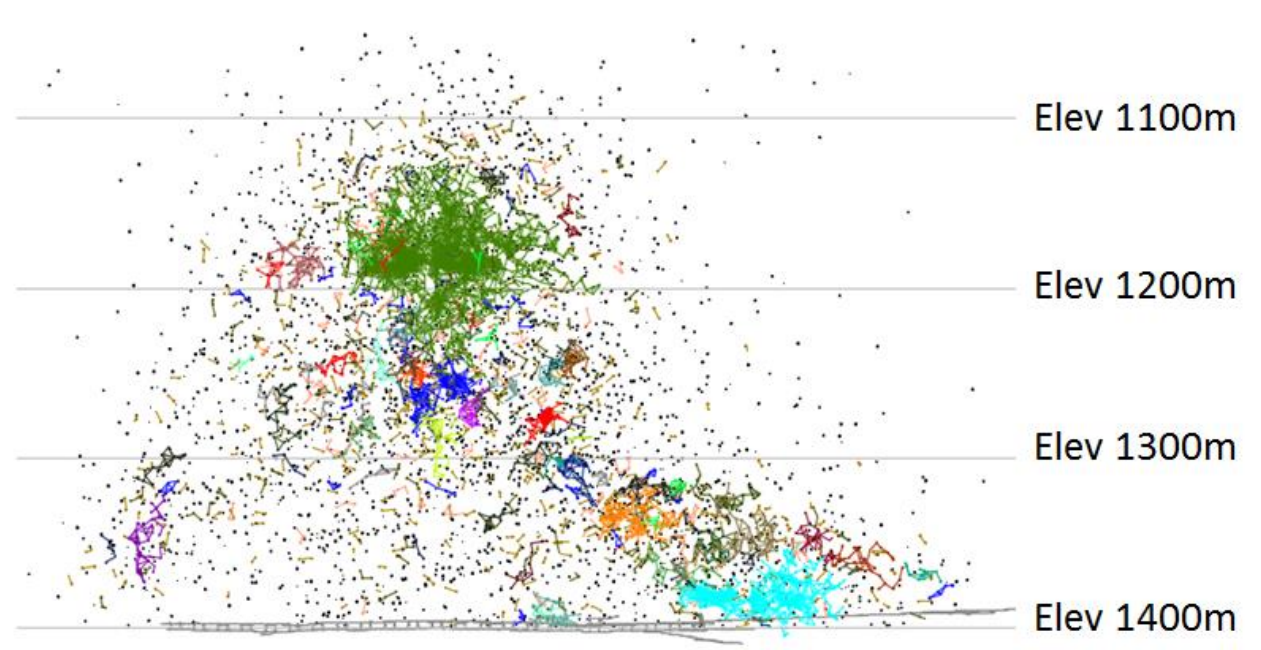

Figure 9 All clusters formed during nine months as the cave initiated. The mean nearest neighbour distance of $7.8 \mathrm{~m}$ is used to cluster the events

The different cluster sizes and locations are useful to identify which areas of the rock mass are seismically active. In addition, the clusters can be analysed separately to determine if rock mass failure at one location is different than another. This provides the ability to assess seismic hazard in different locations within or around the cave.

The last phase (one month of the propagation of the cave) has an average nearest neighbour distance of $7.0 \mathrm{~m}$. The size and number of clusters created are shown in Figure 10.

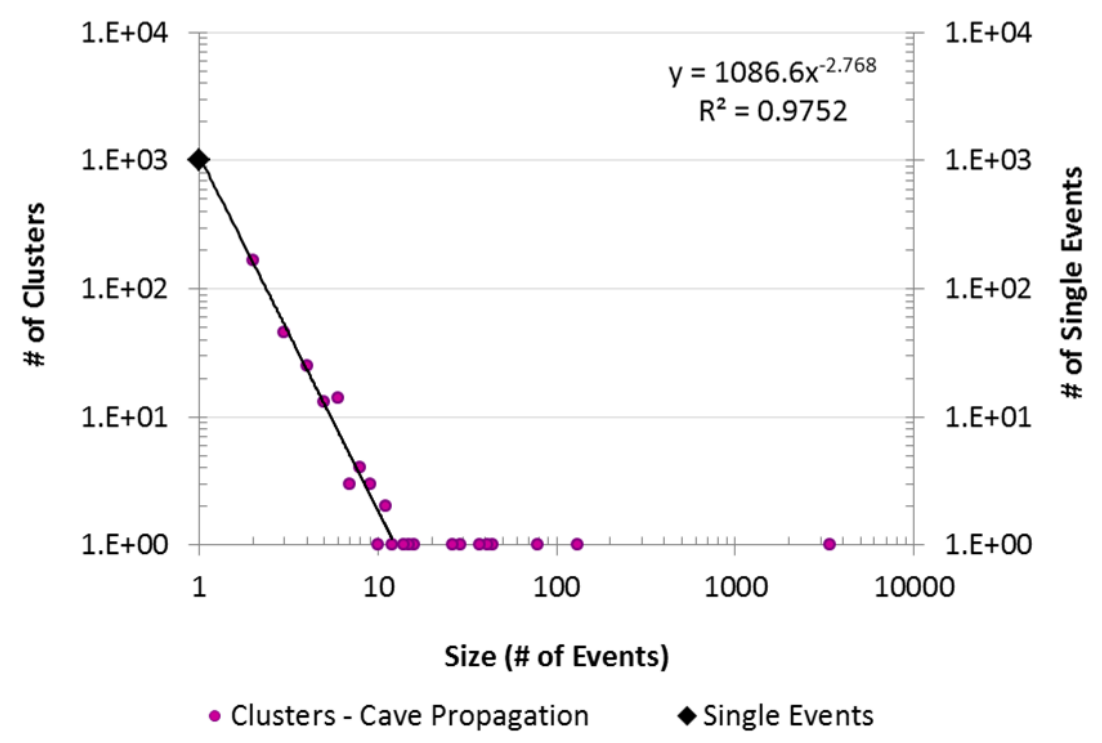

Figure 10 Cluster sizes formed during the propagation of the cave. This period of seismic activity differs from the other two in that there is one large cluster of 3,398 events with all other clusters at least 30 times smaller than the main cave cluster 
As noticed with the other two phases, the propagation phase of the cave is again distinctly different. The largest cluster is now more than 30 times larger than the next nearest event. This cluster contains the events that are in the main cave propagation zone. Fewer events and clusters are occurring just above the undercut (Figure 11).

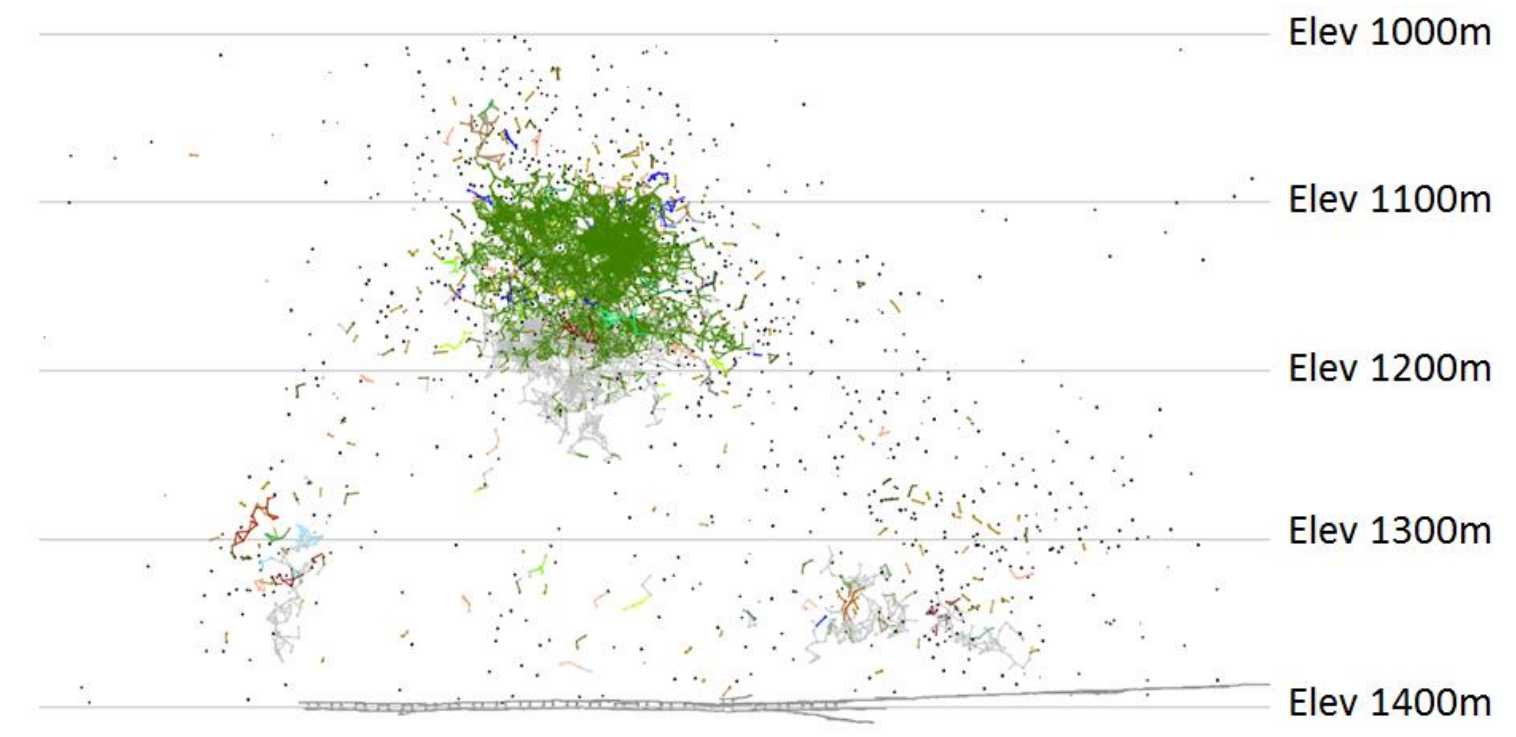

Figure 11 Cluster sizes formed during the propagation of the cave. This period of seismic activity differs from the other two in that there is one large cluster of 3,398 events with all other clusters approximately 30 times smaller than the main cave cluster

This paper is limited in space and only covers three timeframes to demonstrate the sequential spatial clustering method. In practice, the analysis of cluster size, shape and progression should take place as each event occurs. The results could then be compared to a theoretical model. The differences between the two could possibly be used to indicate if the caving rate is too slow, the direction of the cave is changing in an unfavourable direction, and/or the cave is not progressing in one area. This work is beyond the scope of this paper but shows the possible uses of the technique and where research needs to continue.

\subsection{Characterisation using fractal dimension}

The seismic events in each phase of this study will also be characterised by the fractal dimension of the time between the nearest neighbour events and the magnitude ranges (Richter) by nearest neighbour distance. To interpret the results, it is important to know that the higher the fractal dimension, the more highly correlated the nearest neighbour events are for the parameter being studied (Cortolezzis 2018).

\subsubsection{Time between nearest neighbours}

Since nearest neighbour events are located in close proximity to each other, the fractal dimension of the nearest neighbours in a location can be used to characterise the seismic source. In the case of the undercut development, four fractal periods were evident (Figure 12). The first fractal period is the most highly correlated (fractal dimension of 0.54 ) for nearest neighbours that occur within 2.6 seconds to 8.5 minutes of each other. This short response time is indicative of a rock mass responding to localised blasting. However, the rock mass continues to fracture in the undercut during the other three fractal periods. 


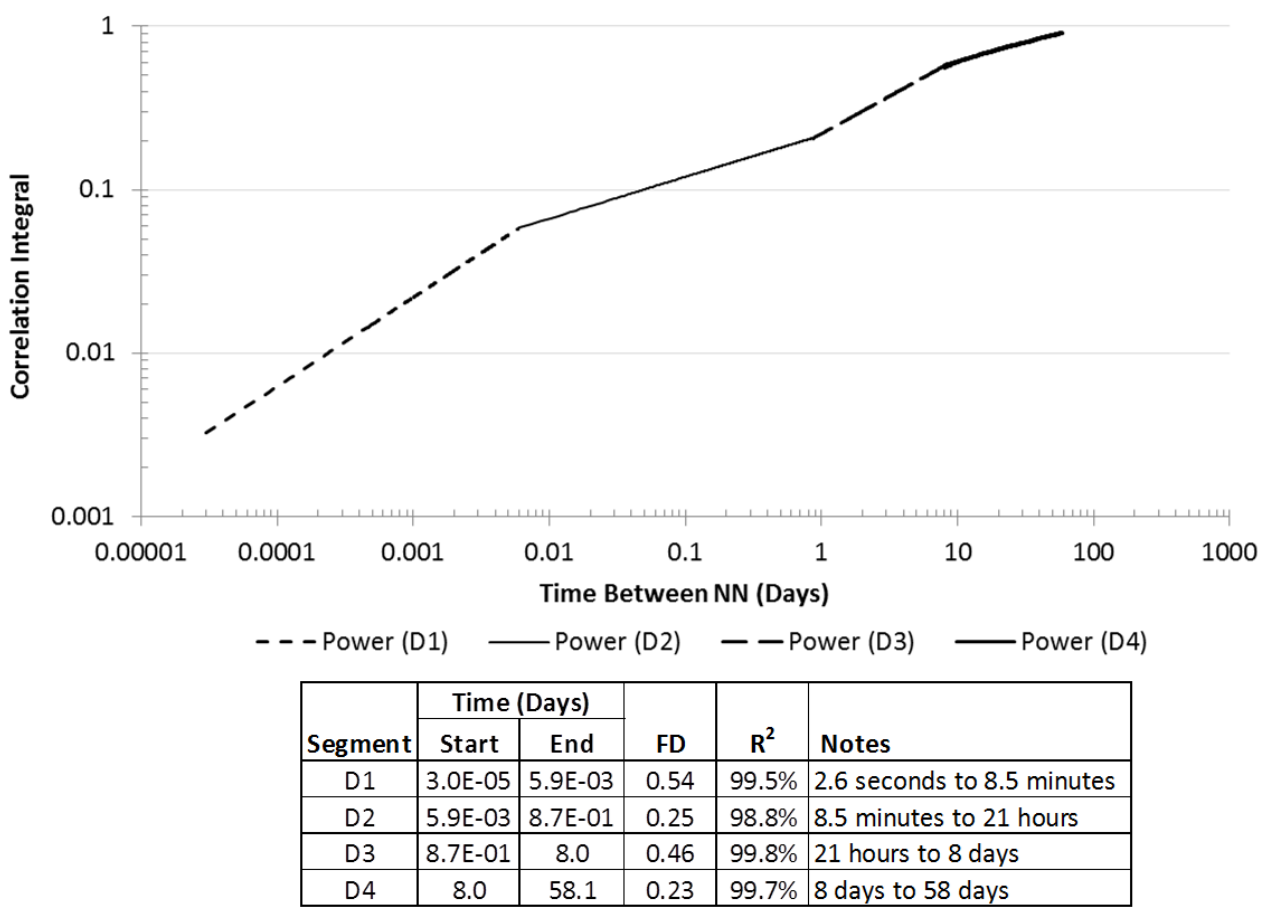

Figure 12 The fractal dimension of time between nearest neighbour events during the development of the undercut. Four fractal periods are noted

The time frame with the highest fractal dimension (highest correlation) in the undercut is during the shortest time frame (2.6 seconds to 8.5 minutes). This is expected in areas being developed using blasting practices. An unexpected result is that the time between nearest neighbour events is fractal (correlated) up to 58 days and not just in the shortest time frames. Further investigation as to the location and time between undercut blasts would be needed to determine if this were a factor in the longer time frame.

The fractal dimension of the nearest neighbour events in the initiation of the cave is well-correlated between eight hours up to five days. However, the remainder of the nearest neighbour pairs are not fractal at all (Figure 13).

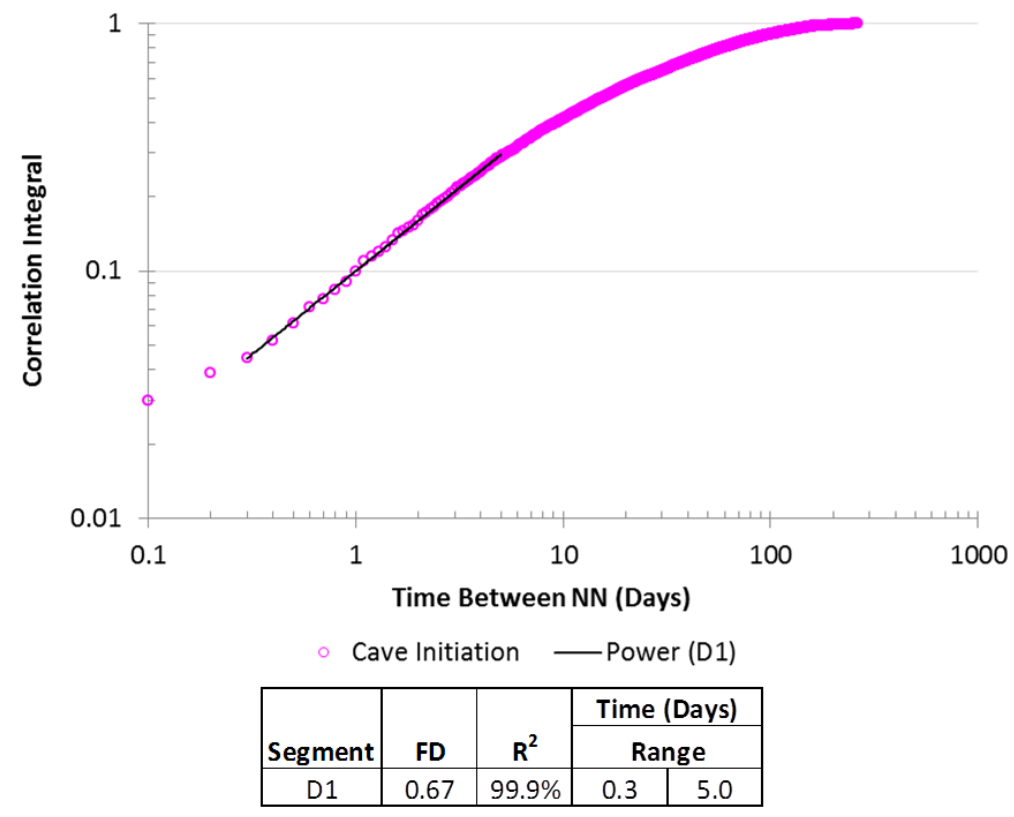

Figure 13 Time between nearest neighbour events during the initiation of the cave. In this phase, only one time period is fractal between eight hours and five days 
The nearest neighbour pairs that are not fractal occur between five and 262 days ( 9 months). The seismic response of the cave initiation is completely different than the undercut. The data shows that there are two different seismic responses occurring. One is mining-driven (undercut) and the other to the mucking rate and cave rate (cave initiation). This indicates that cave initiation is much slower than the undercut phase as a result of the rock mass fracturing without blasting.

The propagation phase of the cave is also fractal for all the nearest neighbour pairs over three different periods. There is a short period that ranges from approximately 2.5 hours to 7 days that is the highest correlated (Figure 14).

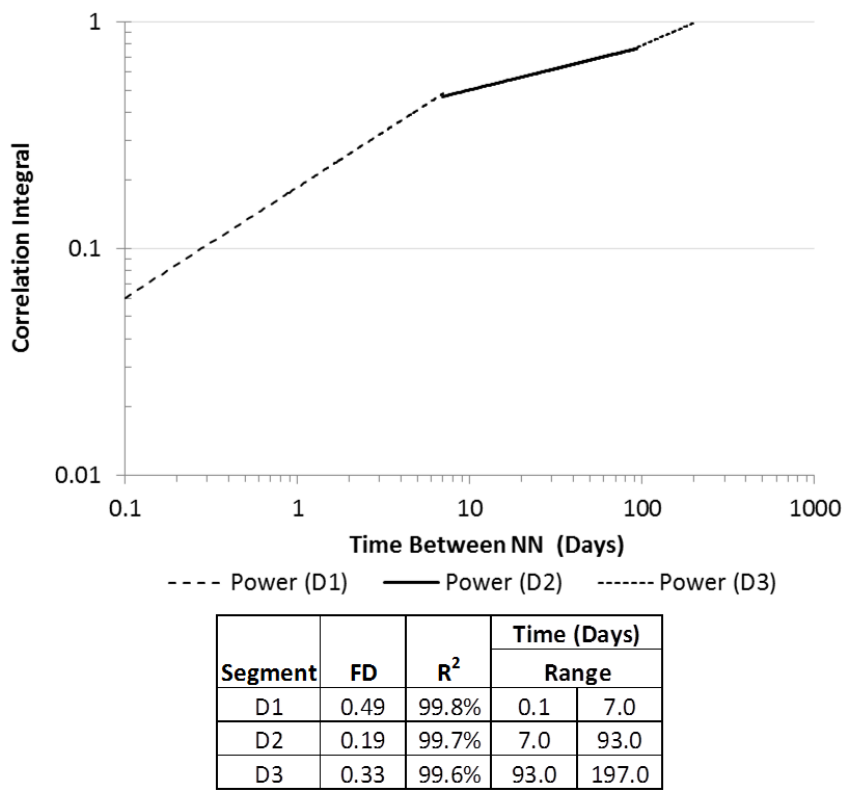

Figure 14 Time between nearest neighbour events during the cave propagation. In this phase, all the nearest neighbour pairs take place in one of three time periods that are fractal

The other two time periods that are fractal are between one week and three months, and then three months to just over six months. The propagation phase is similar to the undercut phase in that the rock mass time periods are all fractal. The difference is that the propagation phase time periods are much longer. They both differ from the initiation phase, which has a very limited fractal period. This is not surprising given that the cave initiation period takes time to establish a void beneath the failing rock mass creating a less consistent rock failure environment. However, it may also be indicative of more than one seismic source. This can be investigated by looking more closely at the clusters within the phase. A visual look at the location of the largest clusters reveals that there appears to be two distinct areas of seismic activity (Figure 15).

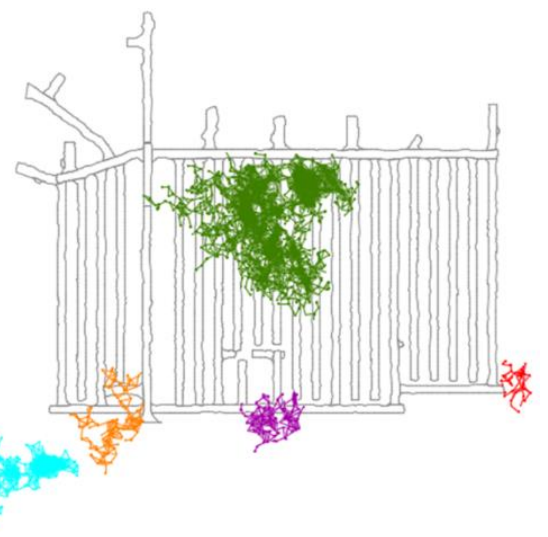

Figure 15 A top-down view of the undercut level and five largest clusters during the cave initiation period. The largest cluster (green) is the initiation of the cave, while the other four clusters look similar to the undercut development phase 
The advantage of the sequential spatial clustering method combined with characterisation is that different locations of seismic activity are mathematically separated and can be studied further to determine if multiple seismic sources are active. Furthermore, the shape of the cave (green cluster) can be compared to a theoretical cave shape to determine if the rock mass is failing where it is expected to.

\subsubsection{Nearest neighbour fractal dimension by Richter magnitude range}

All of the events in each phase are sorted by their magnitude; a measure of intensity. The correlation integral is calculated at each nearest neighbour distance for each magnitude range. The results of this approach show how far apart seismic events can be expected from another seismic event based on their magnitude. This is particularly meaningful to help understand how far the largest magnitude events may occur from existing seismically active locations.

In the case of the undercut area, the largest magnitude events (those greater than Richter magnitude 1) were highly correlated (fractal dimension of 4.7 ) and when they occur 3.5 to $4 \mathrm{~m}$ from another event. The maximum distance a large event occurred was $59.4 \mathrm{~m}$ from another event. Thus, it is reasonable to expect the largest events to occur within close proximity to the undercut (Figure 16).

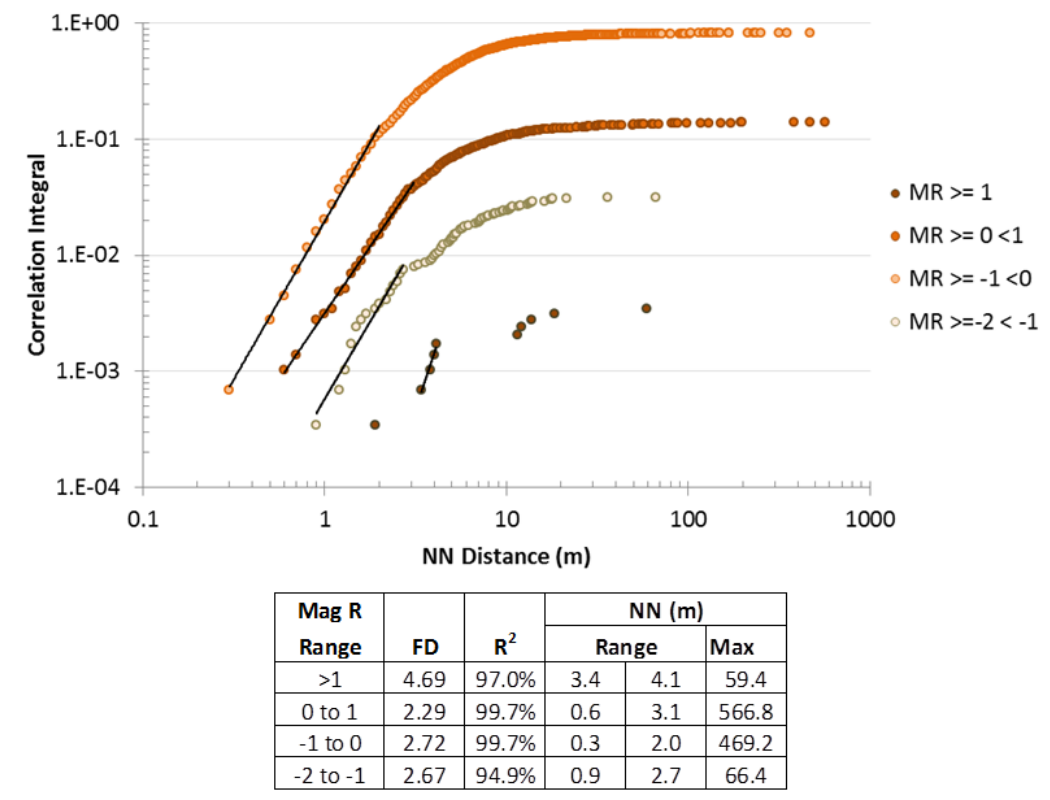

Figure 16 The largest magnitude events (Richter magnitude $\geq 1$ ) are highly correlated and can occur within 3.4 to $4.1 \mathrm{~m}$ from another event. The largest events that are not fractal occurred at further distances of up to $59.4 \mathrm{~m}$, which is in close proximity to the undercut

While the largest magnitude events are highly correlated and occur within fairly close proximity to the undercut area, it should be noted that the remaining lower magnitude ranges are also fractal and within a few metres of another event. Where they differ is that the lower magnitude events can occur much further away from the undercut. From a hazard assessment perspective, this does not pose the same difficulty as the larger magnitude events. What the lower magnitude events do show is how far away the rock mass can fracture.

The magnitude ranges for the initiation of the cave phase are shown in Figure 17. During this phase, the fractal dimension of the nearest neighbour distances are very nearly the same for all magnitude ranges. They range from a fractal dimension of two to just over three. The difference between this phase and the undercut phase is that the largest events in the cave initiation phase are double the distance from their nearest neighbours than in the undercut phase while having similar fractal dimensions. The smaller magnitude events are similar in their range of nearest neighbour distances as well as their fractal dimensions. What is significantly different is that the largest magnitude events that are not fractal can occur from approximately $225 \mathrm{~m}$ to over $450 \mathrm{~m}$ away. This indicates that the cave initiation zone is 
influencing the fracturing of the rock mass quite substantially, not only in the initiation area, but also further afield. This creates the potential for the rock mass failure as the cave initiates to influence geologic structures (known or unknown) to become seismically active. The location of the clusters and their fractal dimension character may provide early insight into these structures.

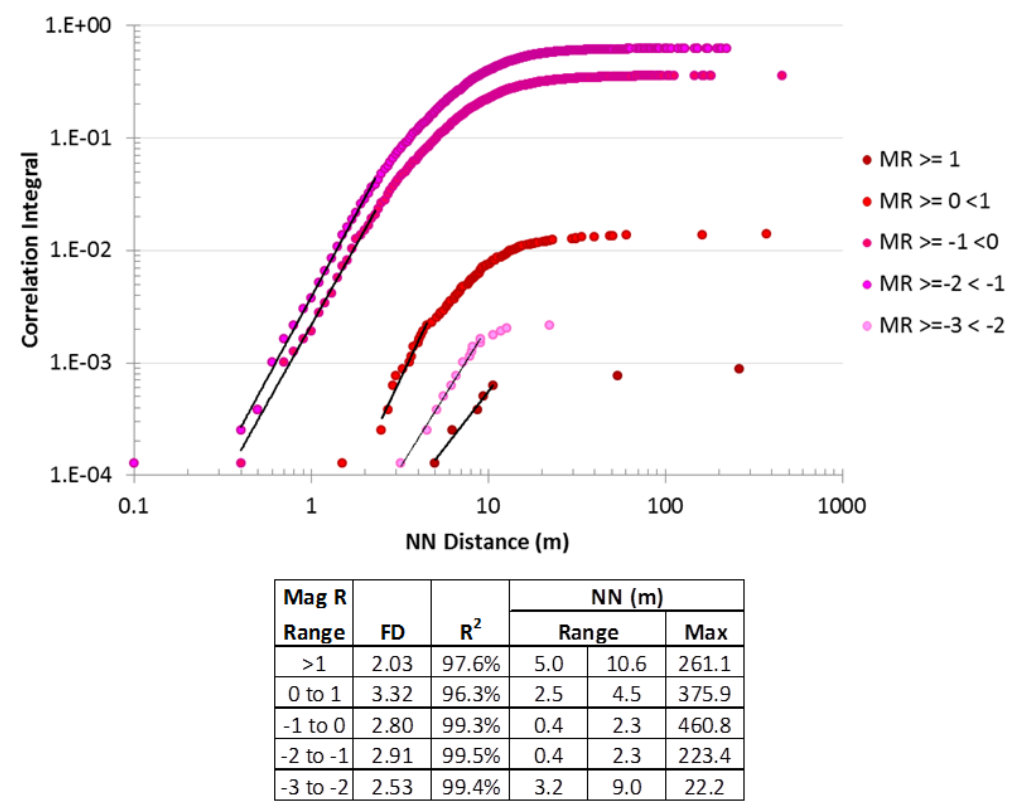

Figure 17 The largest magnitude events (Richter magnitude $\geq 1$ ) in the cave initiation phase are well correlated and can occur within 5 to $10.6 \mathrm{~m}$ from another event. The largest events that are not fractal can occur a substantial distance away $(\sim 260 \mathrm{~m})$

The propagation phase of the cave is highly correlated (fractal dimension of 5.6) for events that have a magnitude greater than or equal to zero but less than one. They can occur up to $89 \mathrm{~m}$ away from another event, which is relatively close to the cave zone (Figure 18). The largest events (Richter magnitude $\geq 1$ ) have a similar nearest neighbour range of 4.6 to $11 \mathrm{~m}$, which is very similar to the same range of large events in the initiation phase. However, the propagation phase large events pose less risk as the maximum distance of non-fractal large events is only $38 \mathrm{~m}$ instead of hundreds of metres.

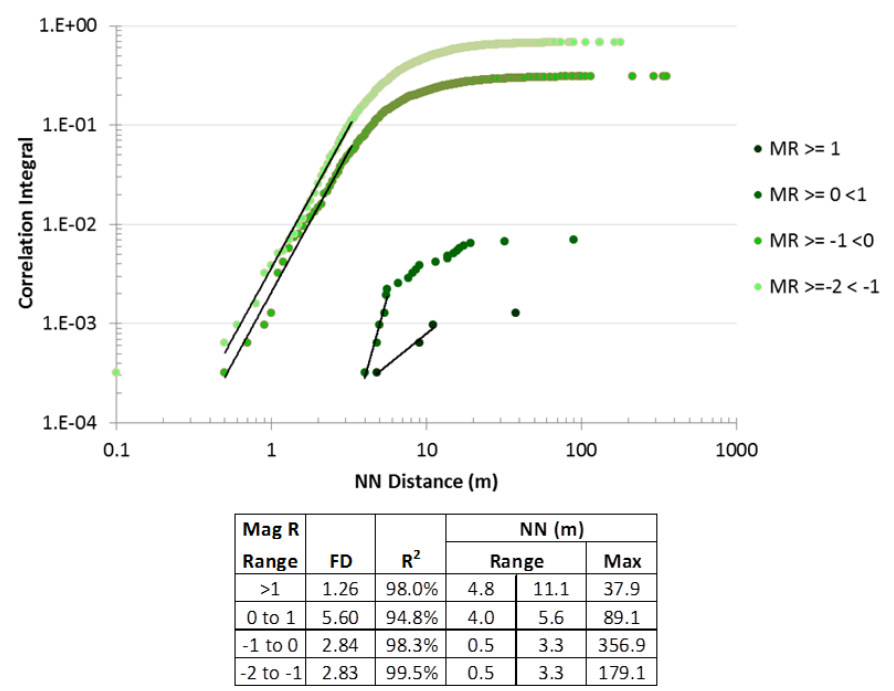

Figure 18 The largest magnitude events (Richter magnitude $\geq 1$ ) are not as well correlated as the lower magnitudes while the cave propagates. In this instance, the largest events can occur within 4 to $11 \mathrm{~m}$ of another event, and no further than $38 \mathrm{~m}$. The events that are less than magnitude 1 but greater than zero are very highly correlated, and are fractal within close proximity to another event, but can occur further away than the largest magnitude events ( $89 \mathrm{~m}$ opposed to $38 \mathrm{~m}$ ) 
All three phases of undercutting the cave, cave initiation, and cave progression have been characterised using the number of clusters and their size, nearest neighbour distances, fractal dimension of the time between nearest neighbours, and the fractal dimension of nearest neighbour distances for all magnitude ranges. The three phases presented in this case study can be used as benchmarks for other cave examples. In particular, the first month of the cave propagation can serve as a comparison for the entire cave as it propagates to surface. Changes in the size and location of the clusters or fractal dimension characteristics of time and magnitude are indicators that the cave propagation is changing. The advantage, of course, is that the changes can be noticed early as the clustering and fractal dimension calculations can be done at any time or with any number of seismic events.

\section{Conclusion}

Sequential spatial clustering has been applied to three phases of a cave: development of an undercut, cave initiation, and cave propagation. The number and size of clusters characterises the seismic response of the rock mass during the phase. In the case of initiation, two seismic sources exist and can be separated by further analysis of individual clusters. Each phase was also characterised by the fractal dimension of the time between nearest neighbour events, as well as the nearest neighbour distances by magnitude range. The ranges of distances that are fractal give a good indication of how far most of the large events occur from existing seismic locations. The large magnitude events that are not fractal have a lower chance of occurring, and the maximum distance these events are from existing events gives an indication of how far they may occur from other seismic events.

Most seismic analysis methods, particularly clustering methods, need large numbers of seismic events and generally take place retroactively. This new type of sequential clustering preserves the order and association each event has with other events that are closest to it. With the order preserved and the relations between nearest neighbours known, clusters of a single seismic source can be studied over time. In this way, clusters created using the sequential clustering method can be used to help identify seismically active areas early and at any point in time and space, particularly as seismicity progresses. This ability to detect unexpected seismically active locations early is a key component to identifying seismic hazards so that proactive mitigation measures can be considered.

\section{Acknowledgement}

Financial support from Laurentian University and the Natural Sciences and Engineering Research Council was instrumental in allowing this research to be conducted and is much appreciated.

\section{References}

Cortolezzis, DM 2018, Characterization of Seismic Sources Using Sequential Spatial Clustering and Fractal Dimension, PhD thesis, Laurentian University, Sudbury.

Coughlin, J \& Kranz, R 1991, 'New approaches to studying rock burst-associated seismicity in mines', in J-C Roegiers (ed.), Proceedings of the 32nd U.S. Symposium Rock Mechanics as a Multidisciplinary Science, A.A. Balkema, Rotterdam, pp. 491-500.

de Beer, W, Jalbout, A, Riyanto, E, Ginting, A, Sullivan, M \& Collins, DS 2017, 'The design, optimisation, and use of the seismic system at the deep and high-stress block cave Deep Mill Level Zone mine', in MR Hudyma \& Y Potvin (eds), Proceedings of the First International Conference on Underground Mining Technology, Australian Centre for Geomechanics, Perth, pp. 233-245.

Digiovinazzo, M \& Singh, U 2010, 'Instrumentation and monitoring of cave initiation at Telfer Mine', in Y Potvin (ed.), Proceedings of the Second International Symposium on Block and Sublevel Caving, Australian Centre for Geomechanics, Perth, pp. 145-155.

Eneva, M \& Young, RP 1993, 'Evaluation of spatial patterns in the distribution of seismic activity in mines: a case study of Creighton Mine, northern Ontario (Canada)', in RP Young (ed.), Proceedings of the 3rd International Symposium on Rockbursts and Seismicity in Mines, A.A. Balkema, Rotterdam, pp. 175-180.

Eneva, M \& Villeneuve, T 1997, 'Retrospective pattern recognition applied to mining-induced seismicity', in SJ Gibowicz \& S Lasocki (eds), Proceedings of the 4th International Symposium on Rockbursts and Seismicity in Mines, A.A. Balkema, Rotterdam, pp. 299-303. 
Glazer, SN 2012, 'Caving process and seismic risk changes, the Palabora Mining Company experience', Proceedings of MassMin 2012, Canadian Institute of Mining, Metallurgy and Petroleum, Westmount.

Hirata, T, Satoh, T \& Ito, K 1987, 'Fracture structure of spatial distribution of microfracturing in rock', Geophysical Journal of the Royal Astronomical Society, vol. 90, pp. 369-374.

Hudyma, MR \& Potvin, Y 2008, 'Characterizing caving induced seismicity at Ridgeway gold mine', in H Schunnesson \& E Nordlund (eds), Proceedings of MassMin 2008, Luleå University of Technology, Luleå, pp. 931-942.

Jones, RH \& Stewart, RC 1997, 'A method for determining significant structures in a cloud of earthquakes', Journal of Geophysical Research, vol. 102, no. B4, pp. 8245-8254.

Kijko, A, Funk, CW \& Brink, AVZ 1993, 'Identification of anomalous patterns in time-dependent mine seismicity', in P Young (ed), Proceedings of the 3rd International Symposium on Rockbursts and Seismicity in Mines, A.A. Balkema, Rotterdam, pp. 205-210.

Kijko, A \& Funk, CW 1996, 'Space-time interaction amongst clusters of mining induced seismicity', Pure and Applied Geophysics, vol. 147, no. 2, pp. 277-288.

Klinkenberg, B 1994, 'A review of methods used to determine the fractal dimension of linear features', Mathematical Geology, vol. 26, no. 1, pp. 23-46.

Lesniak, AJ \& Isakow, Z 2009, 'Space-time clustering of seismic events and hazard assessment in the Zabrze-Bielszowice coal mine, Poland', International Journal of Rock Mechanics \& Mining Sciences, vol. 46, pp. 918-928.

Li, Y, Oh, J, Mitra, R \& Canbulat, I 2017, 'A fractal model for the shear behaviour of large-scale opened rock joints', Rock Mechanics and Rock Engineering, vol. 50, no. 1, pp. 67-79.

Mandelbrot, BB 1982, The Fractal Geometry of Nature, W.H. Freeman and Company, New York.

Pfitzner, M 2003, 'Monitoring a blind sub-level cave - A case study of an integrated approach at Newcrest Mining's Ridgeway Gold Mine', in B Hebblewhite (ed.), Proceedings of the 1st Australasian Ground Control in Mining Conference, UNSW Publishing, Sydney, pp. 113-121.

Pinnock, I, Collins, DS, Toya, Y \& Hosseini, Z 2016, 'Large event sequence analysis and 3D velocity models for seismic event location accuracy', Proceedings of MassMin 2016, The Australasian Institute of Mining and Metallurgy, Melbourne, pp. 879-886.

Potvin, Y \& Hudyma MR 2008, 'Interpreting caving mechanisms using microseismic monitoring data', in H Schunnesson H \& E Nordlund (eds), Proceedings of MassMin 2008, Luleå University of Technology, Luleå, pp. 971-981.

Trifu, Cl, Urbancic, TI \& Young, RP 1993, 'Non-similar frequency-magnitude distribution for M<1 seismicity', Geophysical Research Letters, vol. 20, no. 6, pp. 427-430.

Vejrazka, C 2016, 'Northparkes Mines' current air blast risk assessment practices for block caving operations', Proceedings of MassMin 2016, The Australasian Institute of Mining and Metallurgy, Melbourne, pp. 257-264.

Woodward, K, Wesseloo, J \& Potvin, Y 2018, 'A spatially focused clustering methodology for mining seismicity', Engineering Geology, vol. 232, pp. 104-113.

Xie, H \& Pariseau, WG 1993, 'Fractal character and mechanism of rock bursts', International Journal of Rock Mechanics Mining Sciences and Geomechanical Abstracts, vol. 30, no. 4, pp. 343-350. 